\title{
Sustitución Parcial de Harina de Trigo por Harina de Lupino (Lupinus mutabilis Sweet) en la Producción de Pasta Larga
}

\author{
Mateo Ponce, Danny Navarrete y María G. Vernaza* \\ (1) Universidad San Francisco de Quito - USFQ, Colegio de Ciencias e Ingenierías - El Politécnico, Calle \\ Diego de Robles y Vía Interoceánica, Cumbayá, P.O. Box: 17-1200-841, Quito-Ecuador. \\ (e-mail: mateoponce0192@gmail.com; dnavarrete@usfq.edu.ec; mgvernaza@usfq.edu.ec)
}

${ }^{*}$ Autor a quien debe ser dirigida la correspondencia

Recibido Jul. 27, 2017; Aceptado Oct. 3, 2017; Versión final Oct. 31, 2017, Publicado Abr. 2018

\begin{abstract}
Resumen
Se estudió la sustitución parcial de harina de trigo por harina de lupino (Lupinus mutabilis Sweet) en la producción de pasta larga utilizando metodología de superficie de respuesta. Se utilizó un proceso secuencial de dos diseños experimentales. El primero fue un factorial $2^{2}$ con cuatro puntos centrales. Las variables independientes fueron: cantidad de harina de lupino y huevo. Posteriormente se realizó un diseño central compuesto (DCC). Los resultados en el primer diseño indicaron modelos significativos para la humedad e índice de solubilidad en agua (ISA). EI DCC mostró que la humedad, pérdida de sólidos e índice de absorción de agua (IAA) presentaron modelos significativos. Al aumentar la harina de lupino y huevo en la formulación incrementó el valor de IAA y de pérdida de sólidos. Finalmente, con la función de deseabilidad se determinó la mejor formulación para la obtención de una pasta más nutritiva y de buena calidad con $25 \%$ de harina de lupino y $18 \%$ de huevo.
\end{abstract}

Palabras clave: pasta larga; Lupinus mutabilis Sweet; superficie de respuesta; diseño central compuesto; función de deseabilidad

\section{Partial Substitution of Wheat Flour by Lupine Flour (Lupinus mutabilis Sweet) in the Elaboration of Long Pasta}

\begin{abstract}
The partial substitution of wheat flour by lupine flour (Lupinus mutabilis Sweet) in the elaboration of long pasta has been analyzed using the response surface methodology. Two sequential experimental designs were performed. The first one was a $2^{2}$ factorial design with four central points. The independent variables considered were: the amount of lupine flour and eggs. Subsequently, a central composed design (CCD) was performed. Results from the factorial experiment showed significant models for moisture and water absorption index (IAA). The CCD showed that moisture, solids loss and water absorption index presented significant models. Increasing the lupine flour and egg in the formulation increased the value of IAA and the loss of solids. Finally, with the desirability function was determined the best formulation to obtain a more nutritious and good quality pasta with $25 \%$ of lupine flour and $18 \%$ of egg.
\end{abstract}

Keywords: pasta; Lupinus mutabilis Sweet; surface response; central composed design; desirability function 


\section{INTRODUCCIÓN}

El lupino (Lupinus mutabilis Sweet), también conocido como altramuz es un grano con propiedades nutricionales muy importantes, originario de la región andina de Perú, Ecuador y Bolivia y es común su consumo en el Ecuador y en toda la región andina (Johnson et al., 2017). Para ser apto para el consumo humano, deben eliminarse los alcaloides debido a que pueden ser tóxicos, además de proporcionar un sabor amargo al mismo (Villacrés et al., 2003). La afinidad de los alcaloides para disolverse en agua, debido a sus moléculas hidrofílicas, permite el proceso de desintoxicación de las semillas mediante las fases de hidratación, cocción y lavado (Carvajal-Larenas et al., 2014). La lupanina es el principal alcaloide en la semilla amarga del lupino; es una sustancia tóxica, pero en cantidades pequeñas puede tener efectos farmacológicos (Villacrés et al., 2003). El lupino contiene un rango de compuestos bioactivos, es decir, que tienen actividad biológica dentro del organismo, como carotenoides, que son pigmentos orgánicos con funciones antioxidantes. También tiene fitoestrógenos, que son compuestos químicos con propiedades similares a las hormonas (Villacrés et al., 2003).

El lupino puede ser usado para la fortificación del contenido de proteínas en pasta, pan, galletas, ensaladas, salchichas, entre otros y puede ser sustituto de la soya y la leche (Carvajal-Larenas, 2015), además de ser fuente para la obtención de concentrados y aislados proteicos (Johnson et al., 2017). Estudios realizados por diferentes investigadores demostraron que el consumo de comidas basadas en lupino ayuda a reducir los niveles de lipoproteínas de baja densidad (Sirtori et al., 2004), colesterol y triglicéridos (Arnoldi, 2008), y la presión sanguínea (Lee et al, 2009). La harina de lupino se puede usar para hacer pan, galletas, pasta y mejorar su contenido proteico, calórico y de fibra. El lupino está compuesto por 35-52\% de proteína, 15-24\% de grasa, $8-16 \%$ de fibra, y 2,4-4,2\% de alcaloides (Villacrés et al., 2006, Jayasena y Nasar-Abbas, 2012, Carvajal-Larenas et al., 2016). La harina que se obtiene a partir del lupino es buena fuente de macro y micro nutrientes (Villacrés et al., 2003). El lupino, al contrario de los cereales, es una fuente rica en lisina, aminoácido esencial limitante en productos a base de cereales como el pan y las pastas (Delcour y Hoseney, 2013).

El gluten se forma con la hidratación de las proteínas glutenina y gliadina que están en la harina de trigo. Sus características permiten que se forme una masa elástica que tenga cohesión. Además, el gluten mantiene encapsulado al almidón en la pasta y este permanece así durante la elaboración y cocción de la misma (Delcour y Hoseney, 2013). Adicionalmente, en la preparación de pastas y panes, la calidad de la proteína tiene una importancia mucho mayor a su cantidad (Granito et al. 2003). La metodología de superficies de respuesta es una colección de técnicas estadísticas y matemáticas útiles para modelar y analizar problemas en los que las respuestas de interés son influidas por diversas variables independientes y el objetivo es optimizarlas (Myers et al., 2016). Con todas estas herramientas estadísticas se puede alcanzar el objetivo de determinar las cantidades ideales de ingredientes, para la obtención de productos de calidad. Para analizar múltiples respuestas en simultáneo existe un método que es actualmente uno de los más utilizados en la industria. El método encuentra ciertas condiciones que proveen los valores más deseados en la respuesta utilizándose la función de deseabilidad. Funciona primero de forma individual para cada una de las respuestas, y después encuentra una respuesta global (Myers et al., 2016). La función de deseabilidad asigna números entre 0 y 1 , utilizando una función específica para cada variable. El 0 representa lo menos deseado y el 1 representa el valor ideal de la respuesta. Lo que se busca es encontrar la mejor combinación de condiciones que satisfagan todos los objetivos individuales (Myers et al., 2016). Para juntar todas las respuestas, se utiliza una función objetivo simultánea que es la media geométrica de todas las respuestas transformadas. Si una de las respuestas o factores cae fuera de su rango de deseabilidad previamente establecido, toda la función se hace 0 .

De esta forma, el objetivo de este estudio fue analizar la sustitución parcial de harina de trigo por harina de lupino (lupinus mutabilis sweet) en la producción de pasta larga utilizando la metodología de superficie de respuesta y obtener el punto óptimo utilizando la función de deseabilidad para obtener una pasta con mejor calidad nutricional.

\section{MATERIALES Y MÉTODOS}

Se utilizó sémola de trigo Aberdurum (La Moderna Alimentos S.A.), harina de lupino Tunkawan tostado y molido (Cereales Andinos), huevos y agua potable.

\section{Caracterización de las harinas}

Las mezclas de las harinas (trigo y lupino) con huevo fueron caracterizadas según el ISA y IAA. Se utilizó el procedimiento establecido por Anderson et al. (1969) con algunas modificaciones por triplicado. Se pesó $1 \mathrm{~g}$ de muestra (b.s). Se adicionó $10 \mathrm{~mL}$ de agua destilada a temperatura ambiente y se mantuvo durante 30 minutos bajo agitación. La suspensión se centrifugó a 3000 rpm durante 10 minutos. El sobrenadante se 
evaporó en una estufa a una temperatura de $105^{\circ} \mathrm{C}$ durante 4 horas. Los resultados se calcularon mediante las ecuaciones [1] y [2].

$$
\begin{aligned}
& I S A=(M r e / M a) \times 100 \\
& I A A=M r c /(M a-M r e)
\end{aligned}
$$

Donde:

ISA = índice de solubilidad en agua (\%)

IAA = Índice de absorción de agua ( $\mathrm{g}$ de gel/g de muestra)

Mre = Masa del residuo de evaporación $(\mathrm{g})$

$\mathrm{Ma}=$ Masa de la muestra $(\mathrm{g})$, en base seca

$\mathrm{Mrc}=$ Peso del residuo de centrifugación $(\mathrm{g})$.

\section{Diseño Experimental}

Para cumplir con el objetivo del estudio se utilizó un proceso secuencial de diseño de experimentos y la metodología de superficie de respuesta para evaluar el comportamiento de las variables de interés al modificar las variables controladas (Myers et al., 2016). El primer objetivo se centró en obtener los niveles adecuados de los dos ingredientes (harina de lupino $10-40 \%$ y cantidad de huevo $4-24 \%$ ) y validar si la superficie resultante en la región de experimentación tenía forma lineal o presentaba alguna forma de curvatura (Myers et al., 2016). Para esto se utilizó un diseño factorial $2^{2}$ con 4 puntos centrales lo que resultó en un total de 8 corridas en el experimento. La cantidad de agua requerida para cada combinación se calculó buscando tener siempre un $33 \%$ de absorción de agua. Cuando la cantidad de huevo en la mezcla aumentaba, disminuía la cantidad de agua con el fin de mantener la absorción de agua constante, conociendo que el huevo está compuesto de agua en un $75 \%$ aproximadamente (Khan et al., 2017).

Una vez obtenidos los resultados del diseño factorial, se procedió a realizar un nuevo diseño experimental como parte de la secuencia de estudio que tuvo como objetivo conocer la verdadera relación entre los dos factores de diseño y las variables de respuesta. Se decidió utilizar un DCC. Este diseño experimental tuvo cuatro puntos factoriales, cuatro puntos axiales y tres puntos centrales generando un total de 11 corridas. En la Tabla 1 se presenta el detalle de los factores, niveles y rangos de este experimento.

Tabla 1: Descripción de factores, niveles y rangos para el diseño central compuesto con 2 factores y 3 puntos centrales

\begin{tabular}{|l|c|c|c|c|c|}
\hline \multicolumn{1}{|c|}{ Factor de diseño } & $-\alpha$ & -1 & 0 & +1 & $+\alpha$ \\
\hline Harina de lupino (\%) & 0.86 & 5 & 15 & 25 & 29.14 \\
\hline Huevo (\%) & 1.89 & 5 & 12.5 & 20 & 23.11 \\
\hline
\end{tabular}

Los puntos axiales son puntos en los cuales todos los factores son 0 excepto uno, que toma un valor $\alpha=1,414$ (estos valores están codificados). Para este caso, al tener solo 2 factores, en cada punto axial solo uno de los dos es igual a cero. Este diseño busca ajustar la relación entre factores y respuesta a un modelo de segundo orden, estimar coeficientes para el modelo generado, y encontrar la combinación óptima de factores (Myers et al., 2016).

Para evaluar los resultados obtenidos de las múltiples variables medidas se utilizó la función de deseabilidad. Esta toma en cuenta los objetivos deseados, sea maximizar o minimizar cada una de las variables de respuesta. La deseabilidad compuesta determina la forma en que la estructura optimiza un conjunto de respuestas en general. La deseabilidad arroja un puntaje de 0 a 1, siendo 1 la situación óptima; el 0 indica que los resultados no están en los rangos aceptables. El algoritmo de optimización utiliza valores de ponderación para cada variable de respuesta, las cuales fueron asignados según el objetivo establecido para cada una de ellas. Estas ponderaciones tienen valores de 1 al 5 , donde 1 indica importancia nula; 5 indica un peso máximo en la determinación de la deseabilidad (Myers et al., 2016). Se analizaron todos los tratamientos con esta función y a partir de los puntajes se seleccionó un tratamiento para finalmente ser comparado con un control por medio de los análisis de composición proximal.

\section{Elaboración de la pasta}

Las diferentes formulaciones de la pasta, fueron obtenidas al mezclar previamente las harinas en una batidora planetaria marca HOBART (Estados Unidos) por 15 minutos. Una vez mezcladas las harinas fueron colocadas 
en el extrusor a frío marca La Parmigiana D45, (Italia) donde se adicionó la cantidad de huevo y agua según el diseño experimental utilizado para alcanzar un porcentaje de absorción del $33 \%$ y se mezcló por 15 minutos más. Después de este tiempo, se extrusó la masa por una matriz para la obtención de pasta tipo espagueti. Una vez obtenidos los tallarines de $30 \mathrm{~cm}$ de largo, estos fueron colocados en la cámara de secado hasta alcanzar una humedad del $7 \%$.

\section{Caracterización de las pastas}

Las mezclas de las harinas con la respectiva cantidad de huevo descritas en el diseño experimental fueron caracterizadas de acuerdo a su ISA e IAA siguiendo la metodología descrita por Anderson et al. (1969), con algunas modificaciones como se explicó anteriormente. Las determinaciones se realizaron por triplicado.

Las pastas obtenidas fueron caracterizadas de acuerdo a su contenido de humedad siguiendo la metodología 44-01.01 (AACC, 2010) y de acuerdo a la prueba de cocción (tiempo óptimo de cocción, porcentaje de pérdida de sólidos y aumento de peso) siguiendo la metodología 66-50.01 (AACC, 2010). En la prueba de cocción de determinó el tiempo óptimo de cocción (min), aumento de peso (\%) y pérdida de sólidos solubles (\%). Los análisis se hicieron por triplicado.

Una vez analizados los resultados obtenidos en las todas las variables de respuesta, por medio de la metodología de superficie de respuesta y por la función de deseabilidad se identificó la formulación óptima para elaborar una pasta con buenas propiedades tecnológicas y nutricionales. Dicha formulación fue comparada con una pasta control (solo harina de trigo). Las pastas fueron caracterizadas cuanto a su contenido de humedad, grasa, proteínas y cenizas, siguiendo las metodologías descritas por la AOAC (2010). Estos análisis fueron realizados por duplicado. Los carbohidratos fueron calculados por diferencia.

\section{Análisis de los resultados}

Para generar las corridas, el orden de ejecución y el análisis estadístico necesario (ANOVA), se utilizó el software estadístico Design Expert versión 9. La probabilidad de cometer un error tipo 1, fue del 5\%. Los resultados obtenidos en la composición proximal de las pastas fueron analizados estadísticamente utilizándose la prueba $t(p<0.05)$ con la finalidad de determinar diferencias significativas entre las muestras.

\section{RESULTADOS Y DISCUSIÓN}

Los resultados obtenidos del análisis estadístico del diseño factorial $2^{2}$ con 4 puntos centrales reportaron que solo 2 de las 6 variables estudiadas tuvieron modelos significativos: la humedad y el ISA. En ningún modelo se observó curvatura en la respuesta ni tampoco interacción entre factores. Los resultados sugieren que el área de optimización global está cerca de los niveles altos de la harina de lupino y del huevo, aunque este segundo factor parece ser menos significativo.

Tabla 2: Resultados de la tabla de Anova para el diseño factorial

\begin{tabular}{|c|c|c|c|c|}
\cline { 2 - 4 } \multicolumn{1}{c|}{} & \multicolumn{3}{c|}{ Valor- $p$} \\
\cline { 2 - 5 } \multicolumn{1}{c|}{} & Harina de lupino & Huevo & Interacción & Curvatura \\
\hline Tiempo de cocción & 0.4820 & 0.4820 & 0.1393 & 0.0480 \\
\hline Humedad & 0.0015 & 0.7045 & 0.1679 & 0.2445 \\
\hline Pérdida de sólidos & 0.5440 & 0,0600 & 0.0016 & 0.0023 \\
\hline Aumento en peso & 0.3976 & 0.3126 & 0.0505 & 0.0214 \\
\hline ISA & 0.0018 & 0.7314 & 0.0014 & 0.0012 \\
\hline IAA & 0.0665 & 0.3072 & 0.9785 & 0.1418 \\
\hline
\end{tabular}

El resultado de ANOVA para la variable de respuesta humedad mostró que el factor harina de lupino fue significativo (valor-p $<0.05$ ), pero la cantidad de huevo y la interacción entre los dos ingredientes no lo fue (valor- $p>0.05$ ). Además, no se encontró una curvatura significativa, indicando que todos los modelos son lineales.

El resultado de ANOVA para la variable de respuesta ISA indica que la cantidad de harina de lupino y la interacción entre los dos factores fueron significativos. Sin embargo, la curvatura no lo fue. Finalmente, el resultado del ANOVA para las variables de respuesta tiempo de cocción, pérdida de sólidos, aumento en peso e IAA demostró que no existió significancia de los dos ingredientes, su interacción o curvatura. Los modelos matemáticos no fueron significativos en los niveles estudiados. En la Tabla 3 se presentan los resultados del diseño central compuesto. En base a los resultados (Tabla 4) se observa que existen modelos significativos 
para humedad, pérdida de sólidos e IAA. Sin embargo, todos los modelos son lineales y solo hay uno con interacción de dos factores (pérdida de sólidos).

Tabla 3: Resultados obtenidos en el DCC

\begin{tabular}{|c|c|c|c|c|c|c|c|c|}
\hline Tratamiento & $\begin{array}{c}\text { Valor } \\
\text { codificado } \\
\text { lupino }\end{array}$ & $\begin{array}{c}\text { Valor codificado } \\
\text { huevo }\end{array}$ & $\begin{array}{c}\text { Humedad } \\
(\%)\end{array}$ & $\begin{array}{c}\text { Tiempo de } \\
\text { cocción } \\
(\text { min) }\end{array}$ & $\begin{array}{c}\text { Pérdida de } \\
\text { sólidos } \\
(\%)\end{array}$ & $\begin{array}{c}\text { Aumento de } \\
\text { peso } \\
(\%)\end{array}$ & $\begin{array}{c}\text { ISA } \\
\text { (\%) }\end{array}$ & $\begin{array}{c}\text { IAA de gel/g de } \\
\text { (guestra) }\end{array}$ \\
\hline 1 & -1 & -1 & 11.89 & 9.50 & 4.27 & 100.34 & 7.20 & 2.13 \\
\hline 2 & -1 & 1 & 11.06 & 9.50 & 5.50 & 103.92 & 12.68 & 2.32 \\
\hline 3 & 1 & -1 & 11.81 & 10.50 & 8.07 & 114.64 & 12.60 & 2.68 \\
\hline 4 & 1 & 1 & 7.20 & 11.00 & 5.87 & 112.71 & 13.54 & 2.61 \\
\hline 5 & 0 & 0 & 8.07 & 11.00 & 6.54 & 163.32 & 10.41 & 2.44 \\
\hline 6 & 0 & 0 & 11.94 & 9.50 & 5.19 & 164.79 & 10.12 & 2.37 \\
\hline 7 & 0 & 0 & 9.54 & 9.00 & 5.61 & 125.85 & 10.07 & 2.41 \\
\hline 8 & $\alpha$ & 0 & 9.84 & 11.00 & 7.29 & 127.42 & 8.57 & 2.61 \\
\hline 9 & 0 & $\alpha$ & 9.32 & 10.00 & 6.79 & 177.37 & 13.52 & 2.67 \\
\hline 10 & $-\alpha$ & 0 & 12.12 & 10.50 & 4.79 & 171.58 & 13.19 & 2.26 \\
\hline 11 & 0 & $\alpha$ & 12.85 & 10.00 & 5.45 & 118.45 & 8.10 & 2.30 \\
\hline
\end{tabular}

Tabla 4: Modelos obtenidos del DCC para variables de respuesta de la pasta de lupino

\begin{tabular}{|c|c|c|c|c|}
\hline Variables de Respuesta & Modelo & $R^{2}$ & valor $-p$ & Modelo matemáticos ${ }^{1}$ \\
\hline Tiempo de cocción & Lineal & 0.2610 & 0.2982 & N/A \\
\hline Humedad* $^{*}$ & Lineal & 0.5939 & 0.0272 & $=10.54-0.90^{*} \mathrm{~A}-1.31^{*} \mathrm{~B}-0.94^{*} \mathrm{AB}$ \\
\hline Pérdida de sólidos* & Interacción 2do orden & 0.8272 & 0.0181 & $=5.94+0.96^{*} A+0.12^{*} B-0.86^{*} A B$ \\
\hline Aumento en peso & No significativo & $\mathrm{N} / \mathrm{A}$ & $\mathrm{N} / \mathrm{A}$ & $\mathrm{N} / \mathrm{A}$ \\
\hline ISA & Lineal & 0.4620 & 0.0838 & $\mathrm{~N} / \mathrm{A}$ \\
\hline $\mathrm{IAA}^{*}$ & Lineal & 0.8197 & 0.0011 & $=2.44+0.17^{*} \mathrm{~A}+0.082^{*} \mathrm{~B}$ \\
\hline
\end{tabular}

${ }^{*}$ modelos significativos

${ }^{1}$ Modelos matemáticos con variables codificadas: $A=$ cantidad de harina de lupino, $B=$ cantidad de huevo.

EI IAA mide la cantidad de agua absorbida principalmente por el almidón. El ISA mide la cantidad de componentes solubles liberados del almidón. En la calidad de la harina de trigo, es importante conocer tanto la cantidad como la calidad de las proteínas. Por lo tanto, el IAA y el ISA pueden ser utilizados para determinar la calidad de las proteínas y, en consecuencia, la calidad de la harina después de los procesos de extracción.).

La determinación de IAA e ISA en las diferentes mezclas de harinas ayudó a determinar la cantidad de agua necesaria para realizar las diferentes pastas. La absorción de agua en la producción de pastas alimenticias puede variar de $28-35 \%$. Cuando se mezcla el agua con la sémola de trigo, se forman pequeños aglomerados de tamaño homogéneo. El tamaño de estos aglomerados es el resultado de una correcta adición de agua. Si se adiciona el nivel correcto de agua se formará una masa homogénea, mientras que si se adiciona muy poca agua la masa va a tener una textura no deseada (Delcour y Hoseney, 2013). Es por eso que el valor usado en este estudio fue correcto ya que se obtuvo una masa homogénea con buena textura.

Analizando la curva de contorno (Figura 1a) y el modelo matemático (Tabla 4), se observa que al aumentar la cantidad de harina de lupino y la cantidad de huevo en la elaboración de pastas alimenticias, el valor de IAA aumenta. El aumento en la absorción de agua al adicionar harina de lupino a la formulación de pastas alimenticias se debe a la composición química del lupino ya que esta puede aumentar hasta 3 veces su tamaño debido al alto porcentaje de proteínas presente en el mismo (Villacres et al., 2003). Para la obtención de harina de lupino, esta leguminosa pasa por ciertos procesos como son el remojo y la cocción. En la etapa de cocción se mejora la capacidad de absorción de agua debido que elimina componentes como lípidos y polifenoles (Carvajal-Larenas. et al, 2014), además el almidón del lupino (2.99\%) se gelatiniza, el orden molecular es destruido (rompimiento de puentes de hidrógeno) gradual e irreversiblemente y los gránulos pierden su cristalinidad absorbiendo gran cantidad de agua (Hernández-Medina. et al, 2008).

Es importante conocer la humedad de las pastas ya que este es un parámetro que está relacionado con la estabilidad del producto, mientras menos agua esté presente en este, mayor va a ser su vida útil ya que existe menor probabilidad de crecimiento de microrganismos y reacciones enzimáticas. Según la Norma Técnica Ecuatoriana 1375 (2014), una pasta alimenticia seca deberá tener máximo 14\% de humedad. Los valores de humedad encontrados en las diferentes pastas obtenidas se encuentran en un rango de 7.20 a $12.85 \%$, cumpliendo lo estipulado en la NTE. 


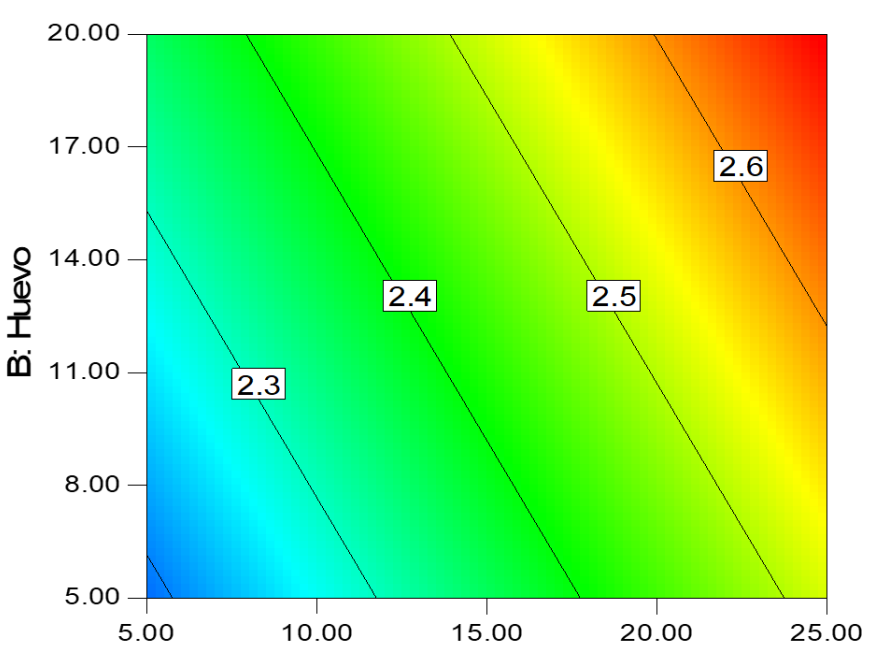

A: Harina de Lupino

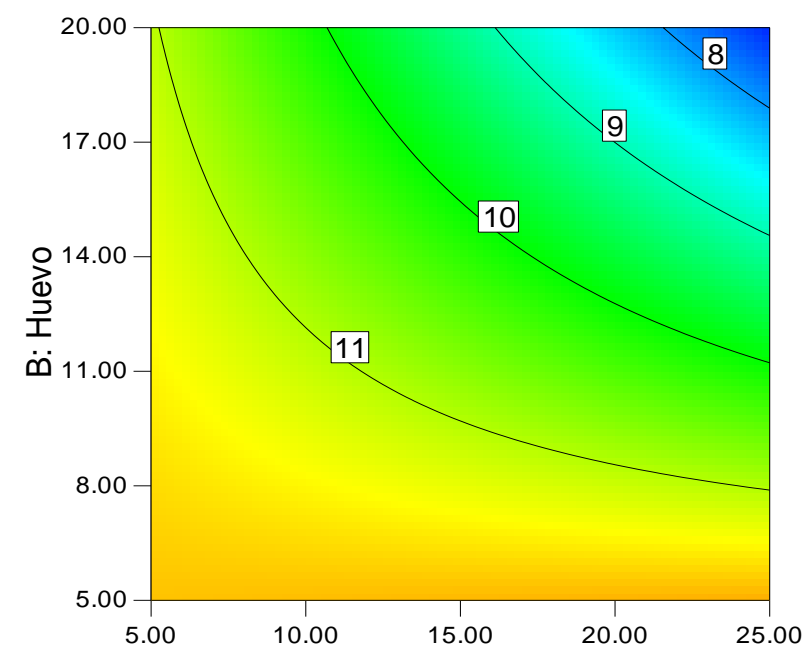

A: Harina de Lupino

b)
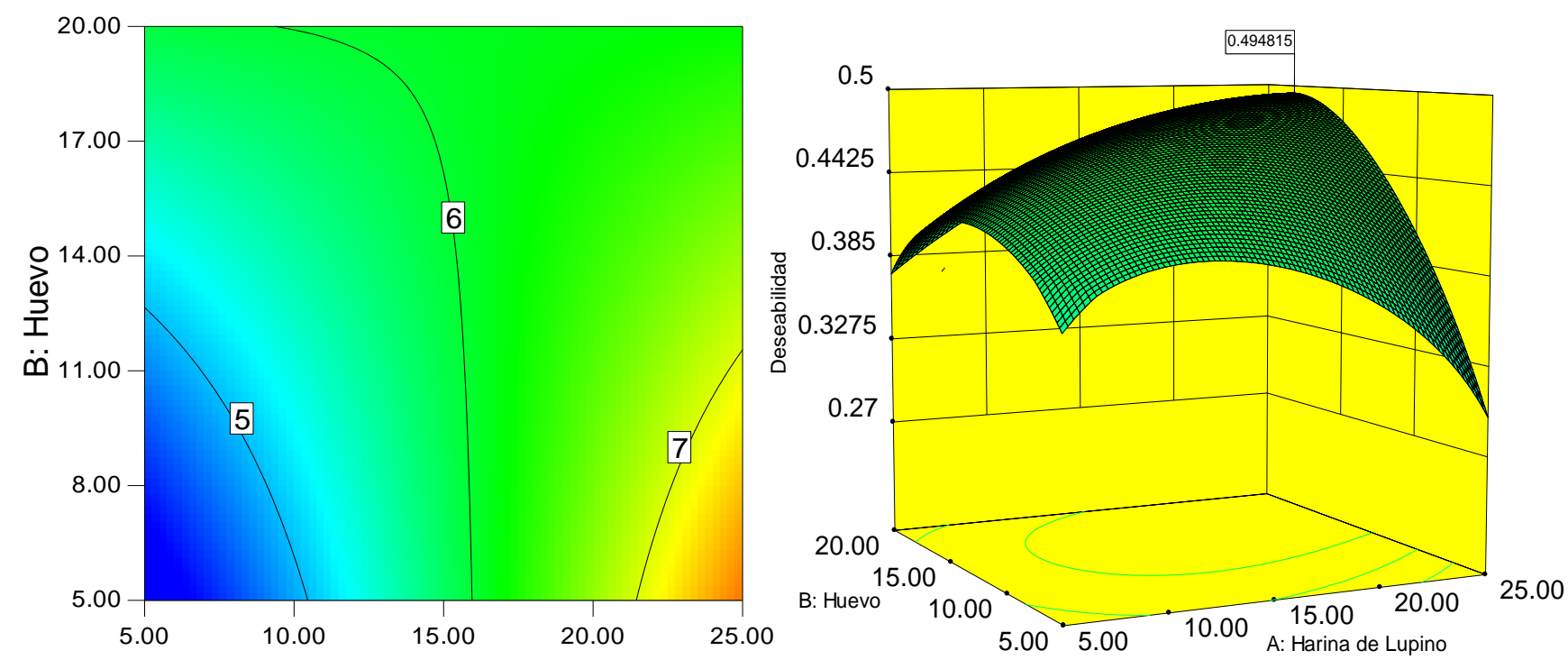

A: Harina de Lupino

c)

d)

Fig. 1: Curvas de contorno de los modelos significativos. a) Curva de contorno de la respuesta IAA, b) Curva de contorno de la respuesta Humedad, c) Curva de contorno de la respuesta Pérdida de Sólidos y d) Superficie de respuesta en 3 dimensiones para la función de deseabilidad.

Analizando la curva de contorno (Figura 1b) y el modelo matemático (Tabla 4), se observa que al aumentar la cantidad de harina de lupino y la cantidad de huevo en la elaboración de pastas alimenticias, el valor de la humedad de las pastas disminuye. Para poder producir pastas alimenticias es necesario añadir una cierta cantidad de agua a la formulación. En este estudio se adicionó 35\% de agua, el cual fue calculado a partir de la cantidad de huevo utilizado. Al momento de secar las pastas, una parte del agua añadido puede quedar ligado a ciertos componentes de los ingredientes. El lupino contiene un $35-52 \%$ de proteína y $8-16 \%$ de fibra (Carvajal-Larenas et al., 2016), los cuales favorecen a la ligación de agua en la elaboración de la pasta.

Cuando se sustituye parcialmente la sémola en la elaboración de pastas alimenticias, podría haber una variación en cuanto a algunos parámetros de calidad como son: el tiempo óptimo de cocción, el incremento de peso y la pérdida de sólidos solubles. El tiempo de cocción es importante porque influye sobre la textura de las pastas. Así, si las pastas se cocinan menos de lo indicado, la textura será dura y resaltará el sabor a harina; si por el contrario las pastas están sobre cocidas, se tornarán blanquecinas, se romperán fácilmente en pequeños trozos y presentarán una textura suave, elástica y pegajosa, lo cual es considerado por los consumidores como una sensación desagradable en la boca (Soo y Baik, 2004). 
La pérdida de sólidos es un indicador para medir la calidad de la pasta cocida. Analizando la curva de contorno y el modelo matemático, se observa que al aumentar la cantidad de harina de lupino y la cantidad de huevo en la elaboración de pastas alimenticias, el porcentaje de los sólidos solubles después de la cocción de la pasta aumenta. En relación a las pérdidas de sólidos por cocción, se ha reportado que la producción de pastas con ingredientes diferentes a la sémola genera un aumento en la pérdida de sólidos, debido a la disminución de la cantidad de gluten presente en las muestras y a la disrupción en la matriz proteica del gluten. Sin embargo, estas pérdidas suelen estar alrededor del $10 \%$, incluso cuando se ha sustituido en un $40 \%$ la harina de trigo por otros ingredientes como harinas de leguminosas, cereales, tubérculos, huevo entero, concentrados y aislados proteicos o mezclas de ellos (Manthey et. Al, 2004). Según Delcour y Hoseney (2013), pérdidas por cocción superiores a $8 \%$ resulta indeseable en la fabricación de pastas alimenticias, alterando la calidad de la pasta. Los valores obtenidos en este estudio son aceptables ya que varían entre el 4.27 y $8.07 \%$ (Tabla 3).

Analizando la curva de contorno (Figura 1c) y el modelo matemático (Tabla 4), se observa que al aumentar la cantidad de harina de lupino y la cantidad de huevo en la elaboración de pastas alimenticias, el valor de la pérdida de sólidos después de la cocción de las pastas aumenta. El gluten se forma con la hidratación de las proteínas glutenina y gliadina que están en la harina de trigo. Sus características permiten que se forme una masa elástica que tenga cohesión. Además, el gluten mantiene encapsulado al almidón en la pasta y este permanece así durante la elaboración y cocción de la misma. El gluten contribuye al desarrollo adecuado de la masa e impide la separación o disgregación de la pasta durante la cocción (Delcour y Hoseney, 2013). Expertos afirman que la sustitución de la sémola de trigo por otras harinas, y la consecuente disminución del contenido de gluten, hace que la calidad de la pasta sea inferior (Cárdenas-Hernández et al., 2016; Lorusso et al., 2017).

Para el análisis multirespuesta, el valor de función de deseabilidad que optimiza las respuestas es de 0,495. Para la obtención de este valor, a cada una de las variables se le asignó un objetivo y un peso (importancia) en consecuencia a lo deseado para la optimización de la formulación final. Las importancias se asignaron entre uno y tres, siendo uno menos importante que tres. De esta forma se asignaron los pesos y objetivos presentados en la Tabla 5. La combinación de factores que permite llegar a este resultado es: $25 \%$ de harina de lupino y $18 \%$ de huevo. Los valores que toman las respuestas en este punto son: tiempo de cocción=10.58 min, humedad $=7.97 \%$, pérdida de sólidos $=6.36 \%$, aumento de peso=137.55 \%, ISA=12.17 \% e IAA=2.66. La Figura $1 \mathrm{~d}$ muestra la superficie de respuesta de la función de deseabilidad en 3 dimensiones.

La combinación óptima está en el nivel alto de la harina de lupino, es decir $25 \%$, y en un punto cercano al nivel alto del huevo, $18 \%$. Se puede afirmar ahora que, al combinar estos factores en estos porcentajes, se obtiene una pasta con índices relativamente apropiados de tiempo de cocción, humedad, pérdida de sólidos, aumento de peso, solubilidad en agua y absorción en agua. Además, se comprobó que la pasta resultante es más nutritiva que la pasta hecha solo con sémola de trigo. La Tabla 6 muestra la composición proximal de la pasta control (solo harina de trigo) y la obtenida con la función de deseabilidad realizada con los niveles altos tanto de harina de lupino como de huevo del diseño central compuesto, reportada como la más óptima según el software utilizado.

Tabla 5: Parámetros utilizados para la obtención de la función de deseabilidad

\begin{tabular}{|l|c|c|}
\hline \multicolumn{1}{|c|}{ Variables } & Objetivo & Peso \\
\hline Harina de lupino & maximizar & 3 \\
\hline Huevo & maximizar & 3 \\
\hline Humedad & minimizar & 3 \\
\hline Tiempo de cocción & minimizar & 1 \\
\hline Pérdida de sólidos solubles & minimizar & 3 \\
\hline Aumento de peso & maximizar & 3 \\
\hline ISA & minimizar & 3 \\
\hline IAA & maximizar & 3 \\
\hline
\end{tabular}

Tabla 6: Composición proximal de las pastas control y óptima

\begin{tabular}{|l|c|c|}
\hline Parámetro & Control $^{1}$ & Óptima $^{2}$ \\
\hline Humedad (\%) & $11,28 \pm 0,17^{\mathrm{a}}$ & $7,61 \pm 0,25^{\mathrm{b}}$ \\
\hline Proteína (\%) & $13,63 \pm 0,52^{\mathrm{b}}$ & $23,77 \pm 0,37^{\mathrm{a}}$ \\
\hline Grasa (\%) & $1,99 \pm 0,47^{\mathrm{b}}$ & $7,94 \pm 0,23^{\mathrm{a}}$ \\
\hline Ceniza (\%) & $1,10 \pm 0,09^{\mathrm{a}}$ & $1,54 \pm 0,3^{\mathrm{a}}$ \\
\hline Fibra (\%) & 0,64 & 1,01 \\
\hline Carbohidratos totales (\%) & 71,36 & 58,13 \\
\hline
\end{tabular}


En la tabla 6, los sub índices indican lo siguiente:

1 Pasta obtenida con $100 \%$ harina de trigo

${ }^{2}$ Pasta obtenida con $25 \%$ de harina de lupino y $18 \%$ de huevo

Medias con diferentes letras en la misma fila indican diferencia estadística entre las muestras por prueba $t$ $(p<0.05)$

La pasta con harina de lupino presentó mejores características nutricionales que la pasta de control (solo harina de trigo). Al aumentar $25 \%$ harina de lupino y $18 \%$ de huevo a la formulación de pasta, la cantidad de proteína aumenta aproximadamente un $43 \%$. El lupino es considerado una leguminosa con alto contenido de proteína (33.9-43.3 g/100 g b.s.) (Jayasena y Nasar-Abbas, 2012; Carvajal-Larenas. et al., 2014), además el lupino presenta un perfil relativamente bueno de aminoácidos con cantidades relativamente altas de arginina $(4.1-11.2 \%)$, leucina $(7.5-9.4 \%)$, lisina $(4.3-5.2 \%)$ y fenilalanina (3.0-6.8\%), sin embargo, presenta cantidades deficientes en los aminoácidos metionina y cisteína (Van de Noort, 2017), los cuales son complementados al usar un cereal (Delcour y Hoseney, 2013). El trigo por otro lado presenta cantidades relativamente altas de metionina y cisteína, que, al ser procesados juntamente con una leguminosa, el producto final puede presentar cantidades adecuadas de los aminoácidos esenciales. De igual manera el porcentaje de minerales es mayor en un $40 \%$ por el alto contenido que presenta esta leguminosa en calcio $(1.5-2.2 \mathrm{~g} / \mathrm{kg})$ ), en fósforo (3.0-5.1 g/ $/ \mathrm{kg})$, en magnesio (1.4-2.1 g/ $/ \mathrm{kg})$ y en potasio $(8.1-9.8 \mathrm{~g} / \mathrm{kg})($ Van de Noort, 2017). El lupino contiene alto nivel de fibra $(8-16 \mathrm{~g} / 100 \mathrm{~g})$ (Carvajal-Larenas et al., 2016) por esta razón la pasta con harina de lupino aumenta en $58 \%$. El aceite de lupino contiene $75 \%$ de ácidos grasos insaturados y el proceso de desamargado no afecta significativamente a este contenido (Carvajal-Larenas. et al, 2014, Van de Noort, 2017). Los ácidos grasos insaturados presentes en mayor proporción en el lupino son el oleico: (C18:1), el linoleico (C18:2) y el linolenico (C18:3) (Van de Noort, 2017).

\section{CONCLUSIONES}

Los resultados obtenidos en el diseño factorial $2^{2}$ con 4 puntos centrales reportaron que solo 2 de las 6 variables estudiadas tuvieron modelos significativos: la humedad y el ISA. Para el DCC se observó que existieron modelos significativos para humedad, pérdida de sólidos e IAA, todos los modelos son lineales. En estos modelos fue observado que el factor que más influyó en las variables estudiadas fue la adición de harina de lupino. Al aumentar la harina de lupino y huevo en la formulación de pasta seca aumentó el valor de IAA y el de pérdida de sólidos solubles. Finalmente, se utilizó la función de deseabilidad para optimizar todas las respuestas en simultáneo. Se determinó que la mejor formulación para la obtención de una pasta más nutritiva y de buena calidad, se consigue con una sustitución de $25 \%$ de harina de lupino y con un $18 \%$ de huevo en la mezcla.

\section{REFERENCIAS}

AACC, Method 66-50.01, Semolina, Pasta and Noodle Quality, Minneapolis, USA (2010)

Anderson, R., Conway, H., Pheiser, V., y Griffin, E., Gelatinization of corn grits by roll and extrusion cooking, Cereal Science Today, 14(1) (1969)

AOAC, Official methods of analysis, Gaithersburg, Md: Associationof Official Analytical Chemists (2010)

Arnoldi, A., Nutraceutical properties of white and narrow-leaved lupin, Actas del 12th International Lupin Conference, Fremantle, Western Australia 14 a 18 de Septiembre (2008)

Cárdenas-Hernández, A., Beta, T., Loarca-Piña, G., Castaño-Tostado, E., Nieto-Barrera, J. O., y Mendoza, S., Improved functional properties of pasta: Enrichment with amaranth seed flour and dried amaranth leaves, https://doi.org/10.1016/j.jcs.2016.09.014, J. of Cereal Sci. 72, 84-90 (2016)

Carvajal-Larenas, F.E., Van Boekel, M.J.A.S., Koziol, M., Nout, M.J.R., y Linnemann, A.R., Effect of Processing on the Diffusion of Alkaloids and Quality of Lupinus mutabilis Sweet, doi 10.1111/jfpp.12105, J. Food Process Pres., 38(4), 14611471 (2014)

Carvajal-Larenas, F.E., Linnemann A.R., Nout, M.J.R. Koziol M. y Van Boekel M.A.J.S., Lupinus mutabilis: Composition, Uses, Toxicology and Debittering, doi 10.1080/10408398.2013.772089, Crit. Rev. Food Sci. Nutr., 56(9), 1454-1487 (2016)

Delcour, J. y Hoseney, C., Principles of cereal science and technology. ${ }^{\underline{a}}$ Ed. pp. 233. AACC International, Minnesota, USA (2013)

Hernández-Medina, M., Torruco-Uco, J.G., Chel-Guerrero, L., y Betancur-Ancona, D., Caracterización fisicoquímica de almidones de tubérculos cultivados en Yucatán, México, http://dx.doi.org/10.1590/S0101-20612008000300031, Food Sci. and Technol. (Campinas), 28(3), 718-726 (2008)

Jayasena, V., y Nasar-Abbas, S.M., Development and quality evaluation of high-protein and high-dietary-fiber pasta using lupin flour, doi 10.1111/j.1745-4603.2011.00326.x, J. texture Stu., 43(2), 153-163 (2012) 
Johnson, S.K., Clements, J., Villarino, C.B.J. y Coorey, R., Chapter 8 - Lupins: Their Unique Nutritional and HealthPromoting Attributes, in Gluten-Free Ancient Grains: Cereals, Pseudocereals, and Legumes: Sustainable, Nutritious, and Health-Promoting Foods for the $21^{\text {st }}$ Century by Taylo, J. y Awika, J., pp 179-221. Woodhead Pub., Duxford, United Kingdom (2017)

Khan, A.A., Kahn, A., Kahn, S., Beg, M.A., Ali, A., Damanhouri, G., Comparative study of fatty-acid composition of table eggs from the Jeddah food market and effect of value addition in omega-3 bio-fortified eggs, https://doi.org/10.1016/j.sjbs.2015.11.001, Saudi J. Biol. Sci., 24(4), 929-935 (2017)

Lee, Y.P., Mori, T.A., Puddey, I.B., Sipsas, S., Ackland, T.R., Beilin, L.J. y Hodgson, J.M., Effects of lupin kernel flourenriched bread on blood pressure: A controlled intervention study, doi 10.3945/ajcn.2008.26708, Am. J. Clin. Nutr., 89, 766-772 (2009)

Lorusso, A., Verni, M., Montemurro, M., Coda, R., Gobbetti, M., y Rizzello, C. G., Use of fermented quinoa flour for pasta making and evaluation of the technological and nutritional features, https://doi.org/10.1016/j.Iwt.2016.12.046, LWT-Food Sci. Technol., 78, 215-221 (2017)

Manthey, F., Saujanya, Y., Dick, T., Badaruddin, M., Extrusion properties and cooking quality of spaghetti containing buckwheat bran flour, https://doi.org/10.1094/CCHEM.2004.81.2.232, Cereal Chem., 81(2), 232-236 (2004)

Myers, R.H., Montgomery, D.C., y Anderson-Cook, C.M., Response surface methodology: process and product optimization using designed experiments. $4^{a}$ Ed., pp. 825. John Wiley \& Sons, New Jersey, USA (2016)

NTE INEN 1375, Pastas alimenticias o fideos secos. Requisitos, 1-9, Quito, Ecuador (2014)

Sirtori, C.R., Lovati, M.R., Manzoni, C., Castiglionl, S., Duranti, M., Magni, C., Morandi, S., D’Agostina, A. y Arnoldi, A., Proteins of white lupin seed, a naturally isoflavone-poor legume, reduce cholesterolemia in rats and increase LDL receptor activity in HepG2 cells, J. Nutr., 134(1), 18-23 (2004)

Soo, C. y Baik, B., Cooking time of white salted noodles and its relationship with protein and amylase contents of wheat, https://doi.org/10.1094/CCHEM.2004.81.2.165, Cereal Chem. 81(2), 165-171 (2004)

Villacrés, E., Gavilanes, K., Díaz, Y., y Peralta, E., Desarrollo del sistema HACCP para una planta de desamargado de chocho (Lupinus mutabilis Sweet) y especificaciones de calidad del grano, Quito, Ecuador (2003)

Villacrés, E., Rubio, A., Egas, L., y Segovia, G., Analítico: Usos alternativos del chocho. Boletín Divulgativo, Quito, Ecuador (2006)

Van de Noort, M. Lupin: An Important Protein and Nutrient Source, in Sustainable Protein Sources, by Nadathur, S., Wanasundara, J. P. D., y Scanlin, L., pp. 165-183, Academic Press, London, UK (2017) 
